\title{
La comprensión e impulso de la Sostenibilidad: un requisito imprescindible para una acción educativa y ciudadana eficaz
}

\author{
Daniel Gil Pérez \\ Departamento de Didáctica de las Ciencias Experimentales y Sociales Universitat de \\ València.daniel.gil@uv.es \\ ORCID: https://orcid.org/0000-0001-6105-5724
}

\author{
Amparo Vilches \\ Departamento de Didáctica de las Ciencias Experimentales y Sociales Universitat de \\ València.amparo.vilches@uv.es \\ ORCID: https://orcid.org/0000-0001-5308-2714
}

[Recibido: 4 Julio 2019. Revisado: 10 Septiembre 2019. Aceptado: 8 Octubre 2019]

\begin{abstract}
Resumen: Diagnósticos concordantes de la actual situación de emergencia planetaria muestran la necesidad de una urgente transición a sociedades que hagan posible una vida justa y digna al conjunto de seres humanos y a las futuras generaciones, al tiempo que se protege la biodiversidad y se revierte la degradación del medioambiente. Esta problemática ha dado lugar a propuestas con distintas denominaciones -transición a la sostenibilidad, decrecimiento, crecimiento inclusivo... - generando controversias que pueden dificultar el establecimiento de una agenda común para hacer frente a los problemas. Proponemos por ello centrar el debate en analizar las medidas que se consideran necesarias, en particular para su impulso desde el campo educativo, para hacer frente al conjunto de dichos problemas y superar así planteamientos nominalistas que provocan confusión en la ciudadanía y dificultan su implicación.
\end{abstract}

Palabras clave: Transición a la Sostenibilidad; Educación para la Sostenibilidad; Límites Planetarios; Decrecimiento; Objetivos de Desarrollo Sostenible.

\section{Contribution of Education to understanding and enhancing Sustainability}

Abstract: Concordant analyses of the present situation of planetary emergency show the necessity of an urgent transition to societies that make possible a fair life to the ensemble of human beings and future generations, while protecting biodiversity and reverting environment degradation. Several proposals have been advanced with this aim -Sustainability Transition, Degrowth, Solidary Growth...generating controversies that difficult the existence of a common Agenda to face the current serious situation. To overcome nominalist debates that confound citizens and hinder their implication, we shall analyse the measures considered necessary to face the ensemble of problems and make possible the transition.

Keywords: Transition to Sustainability; Education for Sustainability; Planetary Boundaries; Degrowth; Sustainable Development Goals (SDG).

Para citar este artículo: Gil-Pérez, D. y Vilches, A. (2019) La comprensión e impulso de la Sostenibilidad: un requisito imprescindible para una acción educativa y ciudadana eficaz. Revista de Educación Ambiental y Sostenibilidad 1(2), 2101. doi: 10.25267/Rev_educ_ambient_sostenibilidad.2019.v1.i2.2101

\section{Introducción: Necesidad de un debate clarificador}

En el número 1 de Revista de Educación Ambiental y Sostenibilidad, García Díaz, Fernández-Arroyo, Rodríguez-Marín y Puig Gutiérrez (2019, p. 2) han propuesto 
"abrir un debate sobre algunas cuestiones que nos parecen claves para el devenir futuro de la Educación Ambiental", expresando su opción "por una educación en y para el decrecimiento como alternativa a la educación para la sostenibilidad". Nos sumamos a esta propuesta de debate, con el propósito, por nuestra parte, de contribuir a clarificar la idea de sostenibilidad desde nuestro campo de trabajo -la educación- para mejor impulsar la resolución de los graves problemas socioambientales a los que nos enfrentamos. Un impulso muy necesario, pensamos, porque la mayoría de los problemas que han conducido a hablar de una situación de emergencia planetaria (Bybee, 1991) han seguido agravándose desde que la comunidad científica y los movimientos ecologistas comenzaran a reclamar, en documentos bien fundamentados -como Nuestro futuro común (CMMAD, 1988)-, la necesidad de poner fin, con urgencia, a un crecimiento depredador e insolidario, absolutamente insostenible en un mundo finito. Y porque la respuesta a esta situación, aunque cada vez mayor, ha sido hasta ahora insuficiente, en particular desde el campo de la educación que aquí nos atañe.

Los diagnósticos de la situación actual (Worldwatch Institute, 1984-2018; Bybee, 1991; Diamond, 2006; FAO, 2018) muestran sobradamente la necesidad de una urgente transición a sociedades que hagan posible una vida justa y digna al conjunto de seres humanos y a las futuras generaciones, al tiempo que se protege la biodiversidad y se revierte la degradación del medioambiente. Esta problemática ha dado lugar a propuestas con distintas denominaciones -transición a la sostenibilidad, decrecimiento, crecimiento inclusivo... - generando controversias que contribuyen a dificultar el establecimiento de una agenda común para hacer frente a la actual situación.

No deja de ser preocupante, efectivamente, que en lugar de las necesarias convergencias se produzcan enfrentamientos entre quienes preconizan opciones supuestamente distintas pero básicamente coincidentes en su rechazo del actual crecimiento depredador e insolidario que está provocando el caos climático, el agotamiento de recursos esenciales y una contaminación sin fronteras que degrada todos los ecosistemas, sin olvidar unas desigualdades inaceptables y una pobreza extrema que afecta a millones de seres humanos. Y es igualmente preocupante que se utilice una misma enseña -sostenibilidad, desarrollo sostenible, o cualquier otra- con significados distintos e incluso opuestos.

Es preciso, pues, clarificar conceptos como sostenibilidad o decrecimiento y evitar incoherencias y enfrentamientos bloqueadores, fruto, como intentaremos mostrar, de malentendidos y de distorsiones -a menudo interesadas- de los conceptos, que provocan el desconcierto de la ciudadanía y perjudican, en definitiva, su necesaria implicación en la construcción de sociedades respetuosas con todos los Derechos Humanos, incluido el derecho a un ambiente saludable.

El estudio de algunos debates que se han venido produciendo con críticas al concepto de sostenibilidad (Sauvé, 2000; García, 2004; Girault y Sauvé, 2008) nos ha llevado a comprender la importancia de superar los enfrentamientos entre quienes, en realidad, coincidimos en lo esencial, es decir, en la necesidad de poner fin a la actual situación de grave degradación socioambiental. Enfrentamientos que están asociados, insistimos, a malentendidos e interpretaciones incorrectas de lo que cabe entender por desarrollo sostenible, decrecimiento, crecimiento inclusivo, etc. (Vilches, Gil y Cañal, 
2010). Consideramos, en efecto, que las controversias y el rechazo mutuo pierden sentido si se procede a analizar y comparar los objetivos y acciones concretas que se proponen para resolver los graves problemas a los que nos enfrentamos, sea cual sea la denominación dada al conjunto de propuestas. En este caso, se pueden apreciar convergencias notables. Ello muestra la necesidad de un mayor rigor y precisión, tanto al presentar la opción propia como, sobre todo, al criticar otras opciones. No se puede, por ejemplo, rechazar la propuesta de desarrollo sostenible (en adelante, DS) -definido como aquel susceptible de satisfacer las necesidades de la generación presente sin comprometer la capacidad de las generaciones futuras para satisfacer sus propias necesidades (CMMAD, 1988)- afirmando simplemente que es preciso "acabar con la palabrería del desarrollo sostenible -ese oxímoron consensual que utilizan los apóstoles de la religión del crecimiento-" (Latouche, 2017, p. 41). Ello constituye una crítica inaceptable, puesto que distorsiona completamente el concepto de DS, introducido precisamente para denunciar la apuesta por el crecimiento indefinido en un mundo finito. Un concepto que parte de la distinción entre desarrollo y crecimiento (Daly, 1997), concibiendo la posibilidad (y necesidad) de mejoras cualitativas o despliegue de potencialidades, sin crecimiento, es decir, sin incremento cuantitativo de la escala física, sin incorporación de mayor cantidad de materiales ni de residuos producidos. Con otras palabras: es el crecimiento lo que no puede continuar indefinidamente en un mundo finito, pero sí es posible favorecer la transición a un DS (Daly, 1997; Meadows, Randers y Meadows, 2006; Sachs, 2008). Una transición posible y necesaria, porque las actuales formas de vida de los seres humanos no pueden continuar y deben experimentar cambios cualitativos profundos, tanto para la mayoría que vive en la precariedad, como para el $20 \%$ que practica un consumo depredador, claramente insostenible. Y esos cambios cualitativos sin crecimiento material pueden permitir avanzar hacia un DS si se diseñan y orientan adecuadamente, ajustando la economía a las exigencias de la ecología y del bienestar social global, es decir, si se orienta la investigación científica y tecnológica, la economía, la acción política y la educación ciudadana al logro de sociedades respetuosas con todos los Derechos Humanos, incluido el derecho a un ambiente saludable.

Cabe insistir en que los conceptos de DS y de sostenibilidad no pretenden sostener -o sea, mantener- lo existente, como parecen interpretar García Díaz et al. (2019, p. 6) cuando escriben "la perspectiva decrecentista cuestiona la noción de sostenibilidad. Es decir itiene sentido hablar de mantener algo cuando estamos en una situación radicalmente desequilibrante de choque con nuestros límites biofísicos?". Tienen razón, no tiene ningún sentido. Ni desde su perspectiva decrecentista, ni desde la que propone una necesaria transición a una sostenibilidad hoy inexistente. Pero esa realidad, sin embargo, no supone cuestionamiento de la noción de sostenibilidad, que como acabamos de recordar, no consiste en mantener algo, sino en lograr una profunda transformación.

Es cierto que, como escribíamos hace una década (Vilches, Gil y Cañal, 2010), es posible, encontrar, incluso en el discurso político y en propuestas sindicales, ejemplos de manejo interesado del concepto de DS como sinónimo de crecimiento sostenido que externaliza sus consecuencias de degradación ambiental, por lo que resulta esencialmente insostenible. Pero esto es algo que debe ser denunciado como tergiversación, en vez de ver en ello la esencia misma del concepto. No tiene sentido, 
pues, que se siga sosteniendo que con el DS "No se pretende limitar el desarrollo (entendido como crecimiento)" (García Díaz et al., 2019, p.3). Si partimos de esta concepción distorsionada de DS será justo atacarla, pero estaremos criticando algo que no es el DS.

Tampoco tiene sentido criticar las propuestas de decrecimiento (Latouche, 2009) como si consistieran en simple ausencia o disminución del crecimiento económico. El mismo Latouche sale al paso de esta interpretación simplista, recordando el desorden que provoca en nuestras sociedades dicha disminución: aumenta la tasa de desempleo y provoca la reducción de los programas sociales, sanitarios, educativos y medioambientales. En realidad, aclara Latouche, el decrecimiento no es más que una enseña tras la cual se agrupan quienes han hecho una crítica radical al desarrollismo. En rigor, añade, sería mejor hablar de acrecimiento, en el mismo sentido que se habla de ateísmo, puesto que se trata de rechazar el culto irracional al crecimiento por el crecimiento. También García Díaz et al. (2019) rechazan cualquier interpretación simplista de lo que denominan decrecimiento, señalando la necesidad de romper con la compartimentación de los saberes y de trabajar con una visión más global e integrada en el sentido del paradigma de la complejidad de Edgar Morin. Algo que les lleva a plantear cuestiones como la necesidad de disminuir la huella ecológica, o la de evitar la superación de los límites planetarios, con las que estamos plenamente de acuerdo.

La educación debe contribuir a evitar estas y otras confusiones, fruto, como ya hemos señalado, de malentendidos y distorsiones, y mostrar las convergencias profundas que el uso superficial de eslóganes (sostenibilidad, DS, decrecimiento, etc.) impide apreciar. El papel fundamental que UNESCO y otras instituciones científicas y educativas han atribuido a la educación en torno a la actual crisis socioambiental planetaria ha sido el de proporcionar a la ciudadanía la formación que permita participar en la adopción de decisiones y puesta en práctica de medidas fundamentadas para avanzar en la construcción de un presente y un futuro satisfactorios (UNESCO, 2014a, 2014b, 2015 y 2017). Insistimos, pues, en la necesidad de evitar debates nominalistas poco rigurosos y escasamente fructíferos y de favorecer la apropiación y puesta en práctica de las medidas consideradas necesarias, tanto por la comunidad científica como por los movimientos sociales y ecologistas, para hacer frente al conjunto de problemas interconectados que amenazan el futuro de la humanidad y afectan ya gravemente a nuestro presente y al de buena parte de la biosfera. Se puede facilitar así el trabajo para la transición a sociedades realmente sostenibles, es decir, favorecedoras de la supervivencia de nuestra especie (García Díaz et al., 2019), con un nivel de vida de calidad para el conjunto de los seres humanos (Gell-Mann, 1992), evitando las desigualdades extremas (origen de conflictos destructivos), y sin poner en peligro a las futuras generaciones y al resto de la biosfera. Se evita así caer en lo que Engelman (2013) denomina acertadamente sosteniblabla, es decir, el uso abusivo y confuso del término sostenible, al que recurren incluso quienes están contribuyendo al crecimiento depredador y también quienes en vez de denunciar el uso distorsionado y engañoso del concepto de DS se dedican a atacarlo.

Esto constituye el objetivo central de este artículo, respondiendo a algunas cuestiones clave como: ¿Qué concepción de transición a la sostenibilidad pretendemos ayudar a 
construir desde la educación? ¿Qué medidas consideramos necesarias para lograr dicha transición? ¿En qué medida estas acciones son convergentes con las propuestas realizadas desde otras perspectivas como la de decrecimiento o la de crecimiento inclusivo? Cuestión esta última fundamental para evitar que debates académicos mal planteados provoquen confusión en la ciudadanía y dificulten su implicación.

\section{¿Qué concepción de transición a la sostenibilidad pretendemos ayudar a construir en la educación? ¿Qué medidas son necesarias para lograr dicha transición?}

La idea de una necesaria transición a la sostenibilidad surgió, como ya hemos señalado, en respuesta a una insostenible situación de emergencia planetaria que amenaza gravemente el presente y futuro de la especie humana y de gran parte de la biodiversidad (Bybee, 1991). Una situación que nos acerca peligrosamente a los límites del planeta -cuyo conjunto constituye un marco donde la humanidad puede desenvolverse con seguridad (Folke, 2013)- e incluso ha superado ya algunos de estos límites, como la concentración atmosférica de gases de efecto invernadero o el ritmo de extinción de especies.

Se ha comprendido que esta situación es fruto, en gran medida, de las actividades extractivas y transformadoras de la especie humana, cuya huella ecológica -es decir, el área de territorio ecológicamente productivo necesaria para obtener los recursos utilizados y para asimilar los residuos producidos- ha superado, globalmente, la biocapacidad del planeta. Se habla por ello de una etapa geológica nueva, el Antropoceno, para destacar la responsabilidad de nuestra especie en el proceso de degradación de la biosfera y, consecuentemente, en la necesaria adopción de medidas fundamentadas para revertir dicho proceso (Gil-Pérez y Vilches, 2017).

Se precisa, pues, una educación que proporcione una percepción correcta de la situación del mundo, profundice en sus causas y, sobre todo, ayude a conocer las medidas que se requieren para hacer frente a los graves problemas interconectados a los que nos enfrentamos y facilite los cambios de comportamiento que permitan su puesta en práctica para avanzar en la transición a sociedades sostenibles. Podemos sintetizar así dichas medidas, cuyo conjunto define, mejor que cualquier otra consideración, la idea de sostenibilidad a la que aspiramos:

- Adoptar estrategias de consumo responsable y economía circular, que reduzcan drásticamente el actual ritmo de agotamiento de todo tipo de recursos esenciales, desde minerales, bosques o bancos de pesca a las reservas de agua dulce y el mismo suelo cultivable. Un ritmo que en la actualidad es muy superior al de la regeneración de dichos recursos, cuando son renovables, o al de su posible sustitución, cuando no lo son, por otros que sí lo sean. Se precisa, por tanto, pasar de un hiperconsumo insostenible de una minoría a un consumo responsable y solidario, accesible a todos.

- Reducir igualmente el ritmo de producción de residuos contaminantes, asociados a la práctica totalidad de las actividades humanas, muy superior en la actualidad al de la capacidad del planeta para digerirlos. Es preciso y posible adoptar medidas, técnicamente ya disponibles, para evitar que una 
contaminación pluriforme y sin fronteras, continúe envenenando suelos, ríos, mares y aire, y afectando a todos los ecosistemas.

- Potenciar la transición energética, es decir, la descarbonización mediante la utilización de energías limpias y renovables, el incremento de la eficiencia de aparatos y procesos (industriales, agrícolas, etc.) y el ahorro energético, para acabar con el acelerado incremento de la concentración de gases de efecto invernadero en la atmósfera, que está provocando un cambio climático visible ya, entre otras muchas consecuencias, en la rápida disminución de las llamadas nieves perpetuas (la más importante reserva de agua dulce con la que cuentan miles de millones de seres humanos) y en el aumento de la frecuencia e intensidad de fenómenos atmosféricos extremos: huracanes, sequías, inundaciones, incendios...

- Remodelar el proceso de urbanización y transformar las ciudades en espacios saludables y sostenibles, evitando su crecimiento desordenado -acompañado del creciente abandono del mundo rural- que potencia los efectos de la contaminación (a causa del transporte, calefacción, acumulación de residuos, etc.) y el agotamiento de recursos (por la destrucción de terrenos agrícolas, el aumento de los tiempos de desplazamiento y consiguiente consumo de recursos energéticos, etc.). Favorecer su conexión con las zonas rurales y vegetación circundante, debidamente protegidas.

- Facilitar los medios que hagan posible una maternidad y paternidad responsables, que programe el número de hijos y el momento de tenerlos (by choice, not by chance, es decir, por elección, no por azar), evitando los numerosísimos embarazos indeseados -particularmente grave para millones de adolescentes- que causan los consiguientes perjuicios para los afectados, amén de provocar el crecimiento explosivo de la población mundial, que puede estar ya cerca de sobrepasar la capacidad de carga del planeta.

- Revertir la acelerada pérdida de biodiversidad, fruto, en buena medida, del crecimiento explosivo de la especie humana (que acapara ya casi tanta producción fotosintética como la totalidad de las restantes especies) y de sus actividades depredadoras. Es preciso poner fin a una sexta gran extinción ahora en marcha, que amenaza con romper los equilibrios de la biosfera y arrastrar a la propia especie humana, causante de esta extinción.

- Proteger igualmente la diversidad cultural y, muy en particular, las culturas campesinas milenarias. No debemos olvidar que la diversidad de culturas es la garantía de una pluralidad de respuestas a los problemas a los que ha de hacer frente la humanidad y que cada cultura no es solo una riqueza para el pueblo que la ha creado, sino un patrimonio de toda la humanidad.

- Poner fin a los desequilibrios insolidarios -insostenibles y éticamente inaceptables- entre una quinta parte de la humanidad impulsada al hiperconsumo depredador y miles de millones de personas que sufren condiciones de vida insoportables que llegan hasta a provocar la muerte por falta de infraestructuras sanitarias e incluso por inanición.

- Se precisa, en definitiva, una transición a sociedades sostenibles, ergo solidarias, que ponga fin al actual sistema socioeconómico, guiado por la búsqueda del máximo beneficio particular a corto plazo y que apuesta por el 
crecimiento económico indefinido en un planeta finito, sin atender a sus consecuencias ambientales y sociales. Ello le convierte en responsable de problemas como los que acabamos de enumerar y de otros igualmente graves, como las dramáticas migraciones y los conflictos y violencias causados por la competitividad, por el afán de controlar los recursos esenciales.

- Avanzar hacia una gobernanza plenamente democrática en todos los niveles, desde el más local al planetario, que incorpore a las legislaciones el respeto y la universalización de las tres generaciones de Derechos Humanos (incluido el derecho a un ambiente saludable) y sustituya la competitiva anteposición de valores e intereses particulares (económicos, étnicos, religiosos, de género...) por la cooperación en beneficio de todas y todos y de las generaciones futuras (Sachs, 2008).

- Favorecer una educación para la sostenibilidad (en adelante, EDS), que proporcione, como ya hemos señalado, una percepción correcta de la situación del mundo, sus causas y medidas que se requiere adoptar, local y globalmente, potenciando así la implicación y la participación ciudadana en la acción política para la toma de decisiones y en la adopción de dichas medidas y el control de los resultados en los diferentes ámbitos: consumo responsable, actividad profesional y acción ciudadana.

Hoy se sabe que estas medidas son todas y cada una de ellas imprescindibles, porque los problemas que pretenden resolver están estrechamente vinculados y se potencian mutuamente, resultando imposible resolver ninguno de ellos sin tener en cuenta los restantes. Así lo explica Diamond (2006, p. 645), tras analizar una docena de graves problemas a los que se enfrentan nuestras sociedades: "Si no resolvemos cualquiera de la docena de problemas sufriremos graves perjuicios (...) porque todos ellos se influyen mutuamente". La ausencia de esta visión global, holística, en la ciudadanía y muy particularmente en los educadores y en los responsables políticos y económicos, constituye un serio obstáculo para la adopción de medidas y acciones realmente efectivas. Ello obliga a incorporar otra medida necesaria para hacer posible la transición a la sostenibilidad:

- Es necesario integrar las aportaciones a la sostenibilidad de las distintas disciplinas científicas, desarrollos tecnológicos, corrientes de pensamiento, movimientos sociales, etc., que han abordado separadamente los diversos problemas socioambientales. Como indican García Díaz et al. (2019), se trataría de sustituir el enfoque atomizado, enciclopédico, aditivo, reduccionista y simplificador. Ello justifica el surgimiento y potente desarrollo de la Ciencia de la Sostenibilidad, fruto de una nueva revolución científica que integra campos de pensamiento y acción hasta aquí inconexos, evitando así que aparentes soluciones a problemas puntuales generen nuevos obstáculos a la transición a la sostenibilidad (Clark y Dickson, 2003; Komiyama y Takeuchi, 2006; Vilches y Gil-Pérez, 2015).

La consideración de objetivos como los aquí resumidos permite precisar qué entender por transición a la sostenibilidad y DS, mejor que un debate más abstracto acerca del significado de sostenibilidad, en el que resulta fácil caer en malentendidos y distorsiones. Y lo mismo sucede, pensamos, con los conceptos de decrecimiento (que debemos interpretar como decrecimiento de la huella ecológica, de las 
desigualdades, de los conflictos destructivos...) o de crecimiento inclusivo (del bienestar del conjunto de la población). Se pueden apreciar así las convergencias entre las propuestas de quienes -con una u otra denominación- se plantean hacer frente a la insostenible situación actual de emergencia planetaria. Por ello mismo, no vemos la conveniencia -y mucho menos la necesidad- de criticar los conceptos de sostenibilidad y DS y proponer nuevas denominaciones. Son conceptos que debido a su utilidad gozan de una amplísima aceptación en la comunidad científica y en la educativa, como evidenció el lanzamiento en 2004 de la Década de la Educación para el Desarrollo Sostenible, el Programa de Acción Mundial de la Educación para el Desarrollo Sostenible (UNESCO, 2014a) o la aprobación por la Asamblea General de Naciones Unidas de los Objetivos de Desarrollo Sostenible y de la Agenda 2030 para su consecución (accesibles en la web de UN/ODS). Las críticas a ambos conceptos son, como hemos intentado mostrar, críticas al uso distorsionado de los mismos y apenas han logrado apoyo académico o social. Nuestro esfuerzo ha ido e irá dirigido a clarificarlos, combatir las distorsiones y, sobre todo, a impulsar la acción educativa y ciudadana para hacer avanzar la transición a sociedades sostenibles.

Naturalmente, no basta con formulaciones genéricas, como promover un consumo responsable o combatir el caos climático. Es necesario precisar y fundamentar las propuestas. Y aunque no podamos extendernos aquí en cada uno de los objetivos enumerados, nos detendremos, a título de ejemplo, en uno de ellos, para ejemplificar la estrecha vinculación de los problemas que caracterizan la actual situación y, por ende, el carácter imprescindible de todas y cada una de las medidas consideradas que deben impregnar la EDS. Hemos elegido para ello la problemática demográfica, a menudo olvidada o erróneamente planteada.

\section{El crecimiento demográfico y su vinculación al conjunto de problemas que debemos superar para la transición a la sostenibilidad}

¿Hasta qué punto está justificada la inclusión del crecimiento demográfico entre los problemas que hay que tener en cuenta para el logro de la sostenibilidad? ¿Cuáles son las razones que se aducen y hasta qué punto son válidas?

La preocupación por el crecimiento demográfico era compartida a finales del siglo XX, y sigue siéndolo a principios del XXI, por numerosos expertos (Ehrlich y Ehrlich, 1994; Diamond, 2006; Engelman, 2012), incluidos los de la Comisión Mundial del Medio Ambiente y del Desarrollo (CMMAD, 1988) y, muy en particular, por el Fondo de Población de las Naciones Unidas (UNFPA), organismo encargado de la salud sexual y reproductiva, una de cuyas misiones es crear un mundo en el que los embarazos que se produzcan sean los deseados.

Resumiremos aquí los argumentos dados por estos y otros autores para justificar su preocupación por el papel que el crecimiento demográfico puede estar jugando, junto a otros problemas socioambientales, en la actual situación de emergencia planetaria (Gil-Pérez y Vilches, 2017):

- A lo largo de la historia del Homo sapiens, su número se ha mantenido muy reducido y hubo que esperar a principios del siglo XIX para que alcanzara los mil millones. Pero en el siglo XX la población se ha más que triplicado. Y 
aunque ahora se esté produciendo un descenso en la tasa de crecimiento de la población mundial, esta sigue aumentando en unos 80 millones cada año, por lo que puede duplicarse de nuevo en pocas décadas.

- Alrededor de un $40 \%$ de la producción fotosintética primaria de los ecosistemas terrestres es usado por la especie humana cada año para comer, obtener madera, leña para hacer fuego, etc. Es decir, la especie humana está próxima a consumir tanto como el conjunto de las demás especies (Diamond, 2006), afectando gravemente a la biodiversidad.

- El consumo de recursos fundamentales por el conjunto de la población supera ampliamente, como ya hemos señalado, su ritmo de regeneración, al tiempo que dicho consumo produce residuos a un ritmo muy superior al de la capacidad de la Tierra para digerirlos. Y todo ello pese a que buena parte de los seres humanos consumen por debajo de lo que exigiría un nivel de vida satisfactorio.

En suma, el reto definitorio del siglo XXI, señala Sachs (2008, p. 17), será afrontar la realidad de que la humanidad comparte un destino común en un planeta superpoblado: "El siglo XXI echará por tierra muchas de nuestras convicciones fundamentales acerca de la vida económica [...] Los desafíos del desarrollo sostenible (preservar el medio ambiente, estabilizar la población mundial, reducir la brecha entre ricos y pobres y poner fin a la pobreza extrema) ocuparán el centro de la escena".

Sin embargo, las fundamentadas llamadas de atención acerca de los peligros generados por la explosión demográfica contrastan con la actual falta de atención generalizada a dicha problemática (Engelman, 2009; Gil-Pérez y Vilches, 2017). Un claro ejemplo de ello es la ausencia de referencias a la demografía al plantear la cuestión de los límites planetarios que, como explica Folke (2013, p. 55), "en conjunto describen un marco donde la humanidad puede desenvolverse con seguridad y que, de respetarse, garantizaría probablemente que la Tierra pueda permanecer en un estado similar al Holoceno". Nueve son los límites planetarios señalados: cambio climático, diversidad biológica, fijación de nitrógeno y de fósforo, reducción del ozono estratosférico, acidificación de los océanos, utilización de agua dulce, cambios en los usos del suelo, contaminación atmosférica por aerosoles y contaminación química. Y aunque se reconoce que "los límites planetarios son interdependientes, pues vulnerar uno de ellos puede hacer que varíen otros límites o que estos sean superados a su vez" (Folke, 2013, p. 60), no se menciona su lógica vinculación con el tamaño de la población humana y los niveles de consumo, es decir, se ignora la absoluta necesidad de hacer frente a la explosión demográfica para evitar la superación de dichos límites.

Pero, como señala Sachs (2008, pp. 219-220):

"Aunque la tasa de crecimiento demográfico del mundo ha descendido, toda complacencia en relación con este tema está fuera de lugar. La población mundial continúa incrementándose a marchas forzadas y en las regiones con menor capacidad para garantizar la salud, la estabilidad y la prosperidad de la población [...] El mundo debería adoptar un conjunto de medidas que contribuyeran a estabilizar la población mundial, mediante decisiones voluntarias, en una cifra en torno a los ocho mil millones de habitantes, en lugar de 
mantener la actual trayectoria que probablemente nos situará en los nueve mil millones o más en el año 2050".

Podemos hablar así de la necesidad de una nueva cultura demográfica, tan necesaria para la transición a la Sostenibilidad como la nueva cultura energética, la nueva cultura del agua, etc.; una cultura demográfica que tenga en cuenta la estrecha vinculación de los problemas y su carácter glocal (a la vez global y local), evitando los planteamientos localistas y a corto plazo, lo que obliga a transformar la pirámide poblacional con muchos más jóvenes que personas ancianas -insostenible porque exige el crecimiento permanente de la población- en aproximadamente un cilindro de crecimiento cero, con números similares de seres humanos en los distintos grupos de edad. Como explica Engelman (2012, pp. 241-242), hay que:

"Adaptarse al envejecimiento de la población en vez de intentar retrasarlo a través de incentivos o programas gubernamentales destinados a aumentar la natalidad [...] Incluso si los actuales responsables de las políticas pudieran incrementar el aumento de la población fomentando índices de natalidad más elevados o la inmigración, tendrían que lidiar con los problemas del envejecimiento en un futuro".

En definitiva, hoy, cuando ya hemos sobrepasado los siete mil quinientos millones de habitantes en el planeta, es necesario centrar la atención en uno de los requisitos fundamentales para poder abordar adecuadamente el problema demográfico: el reconocimiento del derecho humano básico de determinar libre y responsablemente el número y el espaciamiento de los hijos, accediendo a la información y procedimientos de planificación familiar compartida entre mujeres y hombres, así como a los servicios de salud sexual y reproductiva necesarios para poder ejercer este derecho (Engelman, 2012).

Podemos concluir, a la luz de lo discutido hasta aquí, que el problema demográfico incide, ciertamente, en la actual insostenibilidad de las sociedades humanas y debe, pues, ser incorporado en el estudio global de los factores que la afectan, corrigiendo la insuficiente atención que esta relación está recibiendo recientemente (Engelman, 2009).

Por lo que respecta a las propuestas fundamentadas de tratamiento, estas apuntan a lograr que la transición demográfica se produzca y termine lo antes posible, es decir, a conseguir que el número de nacimientos por pareja deje de sobrepasar la tasa de reposición, evitando al propio tiempo que la población se estabilice en cifras superiores a la capacidad de carga del planeta, algo que nuestra civilización, probablemente, no podría resistir. Ello implica que durante un cierto tiempo la pirámide poblacional se invierta y crezca más el número de ancianos que el de jóvenes. Es más, puede llegar a ocurrir que el número de muertes llegue a superar provisionalmente al de nacimientos y que la población solo se estabilice tras una cierta disminución.

Todo ello plantea, sin duda, serios problemas que deben ser previstos. En cualquier caso, los derivados del envejecimiento poblacional son menores que los que conlleva el crecimiento indefinido de la población -algo absolutamente insostenible- o su estabilización en cifras demasiado elevadas para garantizar la sostenibilidad de los 
recursos y ecosistemas, así como el necesario mantenimiento de la biodiversidad, poniendo fin a las actuales extinciones masivas que, de continuar, arrastrarían consigo a la propia especie humana. En definitiva, el bienestar durable del conjunto de los seres humanos exige que la actual transición demográfica estabilice la población en cifras no muy superiores a las actuales.

Debemos recordar, sin embargo, que este requisito, si bien imprescindible, es totalmente insuficiente porque no existe un problema demográfico que podamos abordar y resolver aisladamente, sino que forma parte de la problemática mucho más amplia y compleja de la actual insostenibilidad de nuestras formas de vida y de la urgente necesidad de transición a sociedades sostenibles. Una transición global que exige numerosas y simultáneas transiciones (Gil-Pérez y Vilches, 2017), además de la transición demográfica a la cual nos hemos referido aquí, a título de ejemplo, para mostrar la estrecha vinculación del conjunto de problemas socioambientales y la necesidad de abordar con detenimiento cada uno de ellos como parte de la EDS.

\section{A modo de conclusión: La educación para la Sostenibilidad y los objetivos de Desarrollo Sostenible, herramientas clave para el impulso de la acción ciudadana}

La grave situación de emergencia planetaria a la que nos enfrentamos exige, como hemos intentado mostrar, multiplicar las acciones desde todos los ámbitos, y muy en particular el educativo, para construir una respuesta de la ciudadanía a nivel mundial que reivindique y contribuya a llevar a cabo un conjunto de transiciones interconectadas, todas ellas imprescindibles, dirigidas en síntesis a conseguir, tal como ya hemos avanzado:

- Un consumo responsable, poniendo fin al actual ritmo de utilización de todo tipo de recursos esenciales, muy superior al de su regeneración, cuando son renovables, o al de su sustitución por otros que sí lo sean.

- Una economía circular, que evite la producción continuada de residuos contaminantes, muy superior en la actualidad a la capacidad del planeta para digerirlos.

- Una transición energética para pasar del uso de los recursos fósiles a las energías renovables, evitando el acelerado incremento de la concentración de gases de efecto invernadero en la atmósfera y océanos.

- Una transición urbana, que ponga fin al proceso de urbanización acelerada y desordenada, que se acompaña del abandono del mundo rural.

- Una transición demográfica que haga posible una maternidad y paternidad responsables, "por elección, no por azar", y reconozca el derecho a una vida afectiva y sexual no obligatoriamente vinculada a la procreación.

- Una transición del antropocentrismo depredador del resto de la biosfera al biocentrismo, para acabar con la acelerada pérdida de biodiversidad.

- Pasar de la imposición de la uniformidad cultural a la protección, impulso y disfrute de su diversidad como alternativa a un consumismo material depredador. 
- Una transición laboral para el logro de puestos de trabajo digno para el conjunto de la ciudadanía, con objeto de evitar desequilibrios inaceptables (Poschen, 2017).

- Una transición que ponga fin a la competitividad guiada por la búsqueda del máximo beneficio particular a corto plazo -que se traduce en crecimiento económico y demográfico depredador $y$ en conflictos destructivospotenciando la cooperación, la paz y la universalización de los Derechos Humanos.

En definitiva, se precisa una EDS que contribuya a la implicación de la ciudadanía en las acciones necesarias para la urgente transición global a sociedades más justas y sostenibles. Podemos recordar que uno de los principales esfuerzos globales para abordar la problemática sociambiental en su conjunto fue la proclamación por Naciones Unidas de la Década de la Educación para el Desarrollo Sostenible 20052014 (DEDS), con el propósito de implicar al conjunto de la ciudadanía en la necesaria y cada vez más urgente transición a la sostenibilidad, solicitando para ello una vez más la implicación de todas las áreas y todos los niveles, tanto de la educación formal como de la no reglada. El informe final de la Década, que fue la evaluación institucional de sus resultados, puso de manifiesto que, aunque quedaba mucho por hacer, era patente la difusión internacional creciente del enfoque propio de la Educación para el Desarrollo Sostenible (UNESCO, 2014a).

De hecho, aunque todavía de manera insuficiente dado el agravamiento de la situación, la institución de la DEDS e iniciativas posteriores han impulsado que la atención a la sostenibilidad esté siendo integrada desde hace años en los diferentes niveles educativos y especialmente en la formación del profesorado. Se pueden encontrar ejemplos de buenas prácticas en numerosos países y en todos los niveles y contextos educativos, como puede verse en el informe del Worldwatch Institute (2017) sobre educación ecosocial: cómo educar frente a la crisis ecológica.

Es importante destacar además que el 25 de septiembre de 2015, la Asamblea General de las Naciones Unidas adoptó la Agenda 2030 para impulsar unos Objetivos de Desarrollo Sostenible (ODS) universales e inclusivos, fruto de una amplísima participación que implicó a instituciones académicas, sindicatos, ONG, movimientos ciudadanos, etc. Este impresionante esfuerzo colectivo condujo a consensuar 17 grandes objetivos, con 169 metas concretas que intentan cubrir el conjunto de problemas interconectados a los que se enfrenta la humanidad, evitando que el olvido de alguno de ellos impida avances reales en su conjunto.

En el Documento Educación para los Objetivos de Desarrollo Sostenible (UNESCO: 2017, p. 1) se señala: "La nueva agenda mundial para el Desarrollo Sostenible 2030 refleja claramente esta visión de la importancia de una respuesta educativa apropiada. La educación está explícitamente formulada como un objetivo independiente en el Objetivo de Desarrollo Sostenible número 4 y, además, metas e indicadores relacionados con la educación también están incluidos en otros ODS. La educación de calidad es, pues, tanto una meta en sí misma como un medio para lograr los otros ODS.

Terminaremos insistiendo en que la transición a sociedades más justas y sostenibles es -cualquiera sea la denominación dada a dicho proceso- una imperiosa necesidad 
para garantizar la continuidad de nuestra especie y unas condiciones dignas de vida para el conjunto de los seres humanos.

\section{Referencias bibliográficas}

Bybee, R. (1991). Planet Earth in Crisis: How Should Science Educators Respond? The American Biology Teacher, 53(3), 146-153.

Clark, W. y Dickson, N. (2003). Sustainability science: The emerging research program, Proceedings of the National Academy of Sciences of the United States of America, 100(14), 8059- 8061.

CMMAD. (1988). Nuestro Futuro Común. Madrid: Alianza.

Daly, H. (1997). Criterios operativos para el desarrollo sostenible. En H. Daly y C. Schutze, Crisis ecológica y sociedad. Valencia: Ed. Germania.

Diamond, J. (2006). Colapso. Barcelona: Debate.

Engelman, R. (2009). Population and Sustainability: Can We Avoid Limiting the Number of People? Scientific American, June 1.

Engelman, R. (2012). Nueve estrategias para no alcanzar una población de 9000 millones. En Worldwatch Institute, La situación del mundo 2012. Hacia una prosperidad sostenible, (Capítulo 9). Barcelona: Icaria.

Engelman, R. (2013). Beyond Sustainababble. En Worldwatch Institute, The State of the World 2013: Is Sustainability Still Possible? New York: W.W. Norton.

Ehrlich, P. y Ehrlich, A. (1994). La explosión demográfica. El principal problema ecológico. Barcelona: Salvat.

FAO (Organización de las Naciones Unidas para la Alimentación y la Agricultura) (2018). El Estado del Planeta. [Proyecto conjunto de 11 volúmenes de El País y la FAO http://www.fao.org/espana/el-pais].

Folke, C. (2013). Respetar los límites del planeta y recuperar la conexión con la biosfera, en The Worldwatch Institute, La situación del mundo 2013. ¿Es aún posible lograr la Sostenibilidad? Barcelona: Icaria.

García Díaz, J. E. (2004). Los contenidos de la Educación Ambiental: una reflexión desde la perspectiva de la complejidad. Investigación en la escuela, 53, 31-52.

García Díaz, J. E., Fernández-Arroyo, J., Rodríguez-Marín, F. y Puig Gutiérrez, M. (2019). Más allá de la sostenibilidad: por una Educación Ambiental que incremente la resiliencia de la población ante el decrecimiento. Revista de Educación Ambiental y Sostenibilidad, 1(1), 1101-15. 1101. doi: https://doi.org/10.25267/Rev_educ_ambient_sostenibilidad.2019.v1.i1.1101.

Gell-Mann, M. (1992). Visions of a Sustainable World, Engineering \& Science/Spring 1992, 5-10.

Gil-Pérez, D. y Vilches, A. (2017). El olvido de la demografía en los estudios de Sostenibilidad. Ápice. Revista de Educación Científica, 1(2), 1-17.

Girault, Y. y Sauvé, L. (2008). L'éducation scientifique, l'éducation à l'environnement et l'éducation pour le développement durable. Aster, 46, 7-30. 
Komiyama, H. y Takeuchi, K. (2006). Sustainability science: building a new discipline, Sustainability Science, 1(1), 1-6.

Latouche, S. (2009). Pequeño tratado de decrecimiento sereno. Barcelona: Icaria.

Latouche, S. (2017). Le Monde Diplomatique en Español. En, Atlas de Economía Crítica. Valencia: Cybermonde.

Meadows, D., Randers, J. y Meadows D. (2006). Los Limites Del Crecimiento30: Años Después (Limits to Growth: The 30 Year Update, Spanish Edition). Barcelona: Galaxia Gutenberg.

Poschen, P. (2017). Trabajo decente, empleos verdes y economía sostenible: Soluciones para el cambio climático y el desarrollo sostenible. Madrid: Plaza y Valdés Editores y OIT.

Sachs, J. (2008). Economía para un planeta abarrotado. Barcelona: Debate.

Sauvé L. 2000. Para construir un patrimonio de investigación en educación ambiental. Tópicos en Educación Ambiental 2(5), 51-69.

UNESCO (2014a). Roadmap for Implementing the Global Action Programme on Education for Sustainable Development.

http://unesdoc.unesco.org/images/0023/002305/230514e.pdf.

UNESCO (2014b). Shaping the Future We Want. UN Decade of Education for Sustainable Development (2005-14). Final Report. http://unesdoc.unesco.org/images/0023/002301/230171e.pdf.

UNESCO (2015). Education 2030. Incheon Declaration and Framework for action for implementation of SDG 4. Paris: UNESCO.

UNESCO (2017). Education for Sustainable Development Goals. Learning Objectives. Paris: UNESCO.

Vilches, A. y Gil-Pérez, D. (2015). Ciencia de la Sostenibilidad: ¿Una nueva disciplina o un nuevo enfoque para todas las disciplinas? Revista Iberoamericana de Educación (RIE), 69(1), 39-60.

Vilches, A., Gil Pérez, D. y Cañal, P. (2010). Educación para la sostenibilidad y educación ambiental. Investigación en la Escuela, 71, 5-15.

Worldwatch Institute (1984-2018). The State of the World. New York: W.W. Norton. [Versiones en castellano, La situación del mundo. Barcelona: Icaria]. 